\section{September 2005}

Valvular Heart Disease: Advanced Surgical and Interventional Therapies AATS First Postgraduate Cousre: September 9-11, 2005, Chicago, Illinois, Fairmont Hotel. Director: Delos M. Cosgrove III, MD; Medical Director: Martin B. Leon, MD. For information: Megan Tainer, Meeting Manager (telephone: 978-9278330; E-mail: mtainer@prri.com).

2nd Cardiosurgical Lugano Meeting: Mini Invasive Approaches in Cardiac Valvular Surgery-State of the Art 2005

Meeting: September 17, 2005, Lugano, Switzerland.Addressforinformation: Annapaola Boschet, Congress Manager, Cardiocentro Ticino, Via Tesserete 48, CH-6900 Lugano, Switzerland (phone: +41 91805 31 92; fax: +41 9180531 67; E-mail: annapaola@cardiocentro.org; Web site:www. cardiocentro.org).

Fourth World Congress on Pediatric Cardiology and Cardiac Surgery Congress: September 18-22, 2005, Buenos Aires, Argentina, Sheraton Buenos Aires Hotel \& Convention Center. Address for information: Congress Secretariat c/o Congresos Internacionales S.A., Lima 355 PB "B"-C1073AAG Ciudad de Buenos Aires, Argentina (telephone: +54-114382-5772; fax: +54-11-4382-5730; Web site: www.congresosint.com.ar/pccs2005; E-mail: pccs2005@congresosint.com.ar).

Announcements of major meetings and other significant activities must be received at least 8 weeks before the desired month of publication. Information will be limited to title of meeting, date, place, and an address to obtain further information. Send announcements to Pamela W. Fried, Editorial Office, The Journal of Thoracic and Cardiovascular Surgery, MCP Hahnemann University, $245 \mathrm{~N}$ 15th St, Mail Stop 496, Philadelphia, PA 19102-1192.

\section{October 2005}

Controversies in the Treatment of Cardiovascular Disease: The Fifth in the Series

Symposium: October 6-7, 2005, Santa Monica, California, the Loews Santa Monica Beach Hotel. Address for information: Promedica International CME, a California Corporation, 2333 State St, Suite 203, Carlsbad, CA 92008 (telephone: 760720-2263; fax: 760-720-6263; E-mail: vamartinelli@promedicacme.com; Web site: http://www.promedicacme.com).

Atrial Fibrillation Summit 2005

Summit: October 14 and 15, 2005, Cleveland, Ohio, The Cleveland Clinic Foundation. Address for information: Center for Continuing Education, The Cleveland Clinic Foundation, CME Department KK31, 9500 Euclid Ave, Cleveland, $\mathrm{OH}$ 44195 (telephone: 216-445-5695; 800-7628173; E-mail: ccfcmedcleveland@ccf.org).

Brigham Cardiac Valve Symposium Symposium: October 27-28, 2005, Boston, Massachusetts, Fairmont Copley Plaza Hotel. Address for information: Norman Shostak, Harvard Medical School-CME, 21 Landmark Center, 2nd Floor West, 401 Park Dr, Boston, MA 02215 (telephone: 617-384-8611; fax: 617-384-8686; E-mail: norman_shostak@hms.harvard.edu).

\section{ACCP: Chest 2005}

Course: October 29-November 2, 2005, Montreal, Quebec, Canada, Montreal Convention Centre. Contact name: Member Services (telephone: 1-800-343-2227 US; 1-847-498-1400 international; Web site: www.chestnet.org).

\section{November 2005}

Third International Meeting of the Cardiac Bioassist Association

Meeting: November 8-10, 2005, Fort Collins, Colorado, Marriott Hotel. Contact information: Eric.Monnet@ColoState.edu (Web site: www.cb2a.org).
The Failing Heart Under Stress: Multifactorial Aspects of Acute and Chronic Heart Failure

Symposium: November 20-22, 2005, Amsterdam, The Netherlands, RAI Congress Center. Address for information: Promedica International, a California Corporation, 7777 Center Ave, Suite 500, Huntington Beach, CA 92647 (telephone: 714-7991617, ext 3; fax: 714-799-1686; E-mail: crussell@promedica-int.com; Web site: http://www.promedicacme.com). 
17th Biennial Congress of Association of Thoracic and Cardiovascular Surgeons of Asia (ATCSA) Congress: November 20-23, 2005, Manila, Philippines, Makati Shangri-la Hotel. Address for information: Avenilo P. Aventura, Sr, MD, Chairman, Organizing Committee, 17th Biennial Congress of Association of Thoracic and Cardiovascular Surgeons of Asia (ATCSA), Congress Secretariat: PATACSI Office/ATCSA Secretariat, 2F, Philippine Heart Centre, East Ave, Quezon City 1100, Philippines (Web site: www.atcsa.org; E-mail: infoAatcsa.org).

\section{December 2005}

Pioneering Techinques in Cardiac Surgery: The Fourth in the Series Symposium: December 1-2, 2005, Leipzig, Germany, the Heart Center. Address for information: Promedica International CME, a California Corporation, 2333 State St, Suite 203, Carlsbad, CA 92008 (telephone: 760-720-2263; fax: 760-720-6263; E-mail: vamartinelli@ promedicacme.com; Web site: http://www. promedicacme.com).

\section{January 2006}

NewEra Cardiac Care 9: Innovation and Technology

Conference: January 6-8, 2006, Newport Beach, California, Four Seasons Hotel Newport Beach. Address for information: Aligned Management Associates, Inc, 793-A Foothill Blvd, PMB \#119, San Luis
Obispo, CA 93405 (telephone: 1-805534-0300; fax: 1-805-534-9030; E-mail: info2006@amainc.com; Web site: www. amainc.com/cardiac_care.html).

\section{February 2006}

CREF 26-The San Diego

Cardiothoracic Surgery Symposium:

Science and Techniques of Perfusion

Conference: February 16-19, 2006, San

Diego, California, San Diego Marriott Hotel \& Marina. Address for information: Aligned Management Associates, Inc, 793-A Foothill Blvd, PMB \#119, San Luis Obispo, CA 93405 (telephone: 1-805534-0300; fax: 1-805-534-9030; E-mail: info2006@amainc.com; Web site: www. amainc.com/cref_cardiothoracic.html).

10th World Congress of the International Society for Diseases of the Esophagus: Pathophysiology, Medical and Surgical Management of Oesophageal Disorders

Congress: February 22-25, 2006, Adelaide, South Australia. Chairs: Glyn Jamieson and John Dent. For information: phone: +618 8274 6060; fax: +61 88274 6000; E-mail: jpower@sapmea.asn.au; Web site: www. sapmea.asn.au/isde.

\section{March 2006}

The Society of Cardiothoracic Surgeons of Great Britain and Ireland 2006 Annual General Meeting: March 1316, 2006, Dublin, Ireland, Citywest Hotel.
Further details and abstract submission online at www.scts.org from September 1, 2005 (closing date midnight November 5, 2005). Contact sctsadmin@scts.org.

\section{April 2006}

The American Association for Thoracic Surgery

Annual Meeting: April 29-May 3, 2006, Philadelphia, Pennsylvania, Pennsylvania Convention Center. Address for information: 900 Cummings Center; Suite 221-U, Beverly, MA 01915 (fax: 978-524-8890; E-mail: aats@prri.com; Web site: www. aats.org).

\section{June 2006}

The 14th Annual Meeting of The Asian Society for Cardiovascular Surgery (ASCVS)

Annual Meeting: June 1-3, 2006, Osaka, Japan, Osaka International Convention Center. Address for information: Secretariat of the 14th ASCVS, National Cardiovascular Center, 5-7-1 Fujishirodai, Suita, Osaka, 565-8565, Japan (telephone: 81-64863-7290; fax: 81-6-4863-7289; E-mail: ascvs2006@iris.ocn.ne.jp).

\section{The Western Thoracic Surgical} Association

Annual Meeting: June 21-24, 2006, Sun Valley, Idaho, Sun Valley Resort. Address for information: 900 Cummings Center, Suite 221-U, Beverly, MA 01915 (fax: 978-524-8890; E-mail: wtsa@prri.com). 